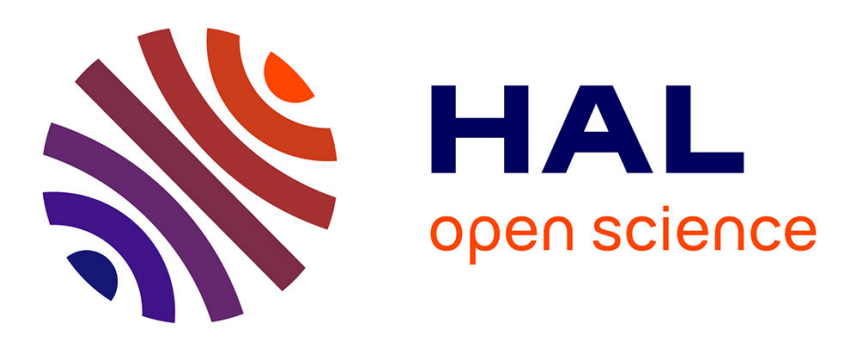

\title{
Ferroelectric volume effect and surface induced fast instabilities for rapid electrooptical displays in liquid crystals
}

Geoffroy Durand

\section{- To cite this version:}

Geoffroy Durand. Ferroelectric volume effect and surface induced fast instabilities for rapid electrooptical displays in liquid crystals. Revue de Physique Appliquée, 1986, 21 (11), pp.669-672. 10.1051/rphysap:019860021011066900 . jpa-00245487

\section{HAL Id: jpa-00245487 https://hal.science/jpa-00245487}

Submitted on 1 Jan 1986

HAL is a multi-disciplinary open access archive for the deposit and dissemination of scientific research documents, whether they are published or not. The documents may come from teaching and research institutions in France or abroad, or from public or private research centers.
L'archive ouverte pluridisciplinaire HAL, est destinée au dépôt et à la diffusion de documents scientifiques de niveau recherche, publiés ou non, émanant des établissements d'enseignement et de recherche français ou étrangers, des laboratoires publics ou privés. 
Classification

Physics Abstracts

$61.30-73.90-68.45$

\title{
Ferroelectric volume effect and surface induced fast instabilities for rapid electrooptical displays in liquid crystals
}

\author{
G. Durand \\ Laboratoire de Physique des Solides, Université de Paris-Sud, 91405 Orsay Cedex, France
}

(Reçu le 20 février 1986, accepté le 23 mai 1986)

\begin{abstract}
Résumé. - Il existe aujourd'hui un système qui permet la commutation rapide à la microseconde, dans les cristaux liquides, sur de la lumière polarisée. C'est la valve $\mathrm{SC}^{*}$ bien connue, qui utilise la ferroélectricité de volume d'un matériau smectique $C^{*}$ chiral et un ancrage de surface faible. Un nouvel effet est présenté, qui donne naissance à des instabilités polaires de surface localisées. Certaines de ces instabilités induites par la surface existent encore pour des excitations alternatives haute fréquence $(1 \mathrm{MHz})$. Bien que leur mécanisme soit encore mal compris, leur temps de réponse, de type nématique, est d'un ordre de grandeur inférieur, à même champ, à celui des SC*. Le contrôle du mécanisme d'excitation par la surface permettrait d'envisager une application électrooptique de ce nouvel effet.
\end{abstract}

\begin{abstract}
One system exist which is capable of microsecond electrooptical switching in liquid crystals, on polarized light. It is the well-known SC* valve, which uses a volume smectic ferroelectric material and a weak surface anchoring. A new effect is presented which gives rise to localized polar surface instabilities. Some of these surface induced instabilities persist for high frequency $(1 \mathrm{MHz}) \mathrm{AC}$ excitation. Their mechanism is not yet understood. Their nematic-like response time is of an order of magnitude shorter, at equivalent field, than the one of the SC*. A control of the surface excitation mechanism would possibly lead to new electrooptical applications.
\end{abstract}

Nematic (N) liquid crystals are now widely used in electrooptical displays [1]. This is due to three properties: 1) their strong coupling with light waves, through their large birefringence, 2) their large sensitivity to external electric fields, because of their large dielectric anisotropy and 3) their weak curvature elasticity which allows large distortions with small fields. The nematic materials for electrooptical applications are now well optimized, to improve the contrast threshold and multiplexability of displays. They are used in well defined texture, oriented by a king of epithaxy from carefully treated boundary surfaces. The corresponding anchoring or the nematic molecules at the surfaces is " strong", i.e. it will not change under the action of the command electric field, during the change of texture which gives rise to the display. One of the major drawback of these nematic display is their long intrinsic response time due to their weak elasticity curvature. Ferroelectric smectic $\mathrm{C}^{*}$ liquid crystals have been proposed to make fast submicrosecond electrooptical switches [2]. Recently, we have demonstrated in Orsay [3] the existence of surface polar instabilities, under the action of an electric field. A more or less distributed surface distortion is observed, with a mechanism related to the ferroelectric character of nematic-solid interface, and to the flexoelectric property of nematics. As SC* this system necessitates weak anchoring conditions to work. Superimposed to the surface flexo-ferroelectric instabilities we have observed other localized surface induced instabilities which can present a fast response time, in the microsecond range. The mechanism of these localized instabilities is not yet fully understood, but their response time seems nematic-like, controlled by the large dielectric anisotropy of the liquid crystal. If well controlled, it could lead to fast electrooptical effects. In this paper, we try to describe the physical problems involved in these fast electrooptic switchings, for both the materials themselves and the associated weak anchoring interfaces. We discuss in particular the threshold effects, related to the multiplexability of these displays. We compare the response time of the bulk $\left(\mathrm{SC}^{*}\right)$ and fast surface induced $(\mathrm{N})$ displays, which turns out to be an order of magnitude more rapid for the same field, at room temperature. The interest of the $\mathrm{SC}^{*}$ ferroelectric system is its linear coupling 
with an external electric field, which allows in principle a bistable behaviour. The interest of fast surface induced display would be its simplicity.

\section{The ferroelectric SC* electrooptic valve.}

The $\mathrm{SC}^{*}$ liquid crystal is a smectic, i.e. a layered material. In each layer, the rod-like organic molecules have no positionnal ordering. There are tilted by an angle $\theta$ compared to the layers (Fig. 1). The molecules are chiral, i.e. they have no more, on the average, the mirror symmetry which characterizes the $\mathrm{N}$ and smectic A phases, because of the presence, for instance, of an asymetric carbon in the molecule. The only symmetry element which characterizes this monoclinic phase is a two-fold flip-flop axis, inside the layers and perpendicular to the long molecular axis. Along this two-fold axis, if there exist any molecular permanent transverse electric dipole, there is a possibility of existence for an averaged macroscopic electric polarization $\mathbf{P}$. The $\mathrm{SC}^{*}$ is then ferroelectric. Of course, if $\theta=0$, the $\mathrm{SC}^{*}$ becomes a $\mathrm{SA}$, with molecules normal to the layers. $\mathbf{P}$ is then zero. At a $S_{A} \rightarrow S_{C}^{*}$ second order transition, $P$ must vary in the $\mathrm{S}_{\mathrm{C}}^{*}$ phase as $\mathbf{P}=\mathbf{P}_{0} \boldsymbol{\theta}$, by symmetry. $P_{0}$ is found to be $10^{-1}$ to $10^{-2}$ of the transverse permanent dipole of the molecule.

The normal texture of a thick $\mathrm{S}_{\mathrm{C}}^{*}$ sample looks like the one of a cholesteric. The chirality of the molecules induces a twist of the projection of $\mathbf{n}$ (the long molecular axis) on the smectic layers, resulting in a heliconical arrangement, with a macroscopic pitch $Z$, in the $\mu \mathrm{m}$ range. It is possible to supress this helix (which is characterized by the observation, across its axis, of dechiralization disclination lines) by putting the sample in between two parallel glass plates, separated by a distance $d<Z$, in the $\mu \mathrm{m}$ range. This is the geometry of the $\mathrm{SC}^{*}$ electrooptical valve proposed by Clark and Lagerwall [2] (Fig. 2). $\mathbf{n}$ is uniform, parallel to the plates. The $\mathrm{SC}^{*}$ layers are

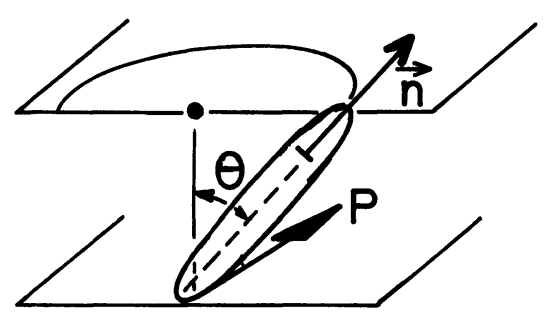

Fig. 1. - Geometry of a smectic $C^{*}$ layer, showing the cone of half-angle $\boldsymbol{\theta}$, and the permanent polarization $\mathbf{P}$.
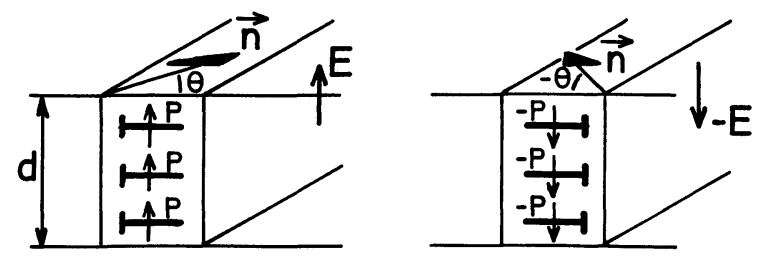

Fig. 2. - Principle of the SC* valve. normal to the plates. $\mathbf{P}$, inside the layers, is also normal to the plates. To provoque a change of texture, it is sufficient to apply a large enough electric field $\mathbf{E}$ across the sample, opposed to $\mathbf{P}$. Above a certain threshold $E_{\text {th }}, \mathbf{P}$ will align along $\mathbf{E}$. n will remain parallel to the plates, but will have rotated of an angle $2 \theta$, remaining on the cone of angle $\theta$ which does not disturb the $\mathrm{SC}^{*}$ ordering. For $2 \theta=45^{\circ}$, using polarized light, in crossed polarizers, a polarized light beam going through the system will go from the total extinction to a total transmission, if the new texture corresponds to a half-wave plate. Calling $\Delta n$ the material birefringence and $\lambda$ the wave length of light, this happens for $d \Delta n \sim \lambda / 2$, easily achieved with $\Delta n \sim 0.2, \lambda=$ $0.6 \mu, d=1.5 \mu \mathrm{m}$. The problem is now to estimate $E_{\mathrm{th}}$, and the response time of the device. In principle, in absence of field, the two states have the same volume energy, so that one expects $E_{\mathrm{th}}$ to be related only to the elastic potential barrier to go from one texture to the other one. In fact, in this device, one must also change the molecular orientation on the two surfaces, which implies a « weak » anchoring surface energy. A complication, discussed by Handschy and Clark [4], is related to the polar character of the anchoring, i.e. at the surface the molecules prefer to align in such a direction that their transverse polarization has a well defined direction compared to the boundary glass electrodes. This allows for the existence of non-uniform textures (Fig. 3), where $\mathbf{P}$ is splayed from one plate to the other. Because of the weakness of the surface anchoring, the surface molecules are no longer restricted to remain parallel to the electrodes. The volume twist (on the smectic $C^{*}$ cone) gives a permanent torque which tilts a little bit the molecules from the surface, resulting in four surface states, with complicated defects between the associated various textures.

The explanation of the rapid (submicrosecond) switching is not yet well understood. We can try to make estimations. As $\mathbf{n}$ is supposed to rotate on the $\mathrm{SC}^{*}$ cone, any volume texture distortion can be described by a nematic-like standard curvature elasticity. The volume elastic barrier density energy is of the order of $K / d^{2}$, with the Frank constant $K$ $10^{-6} \mathrm{cgs}$, which gives a response time of the order of

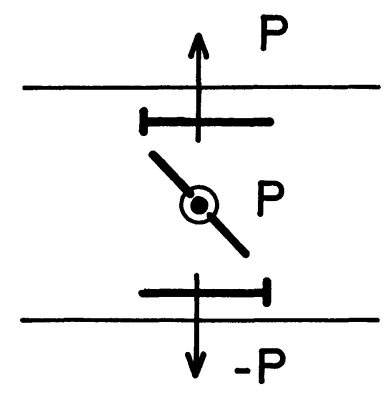

Fig. 3. - The polar anchoring of a SC*. $\mathbf{P}$ is now splayed from the upper to the lower plate. 
$\tau_{\mathrm{N}}=d^{2} \eta / K$, where $\eta \sim 0.1 \mathrm{cgs}$ is a standard viscosity. This time $\tau_{N}$ is a typical nematic like curvature relaxation time, in the $10^{-3} \mathrm{~s}$ range and not in the $\mu s$, so that, at threshold where the coupling electric energy density - E.P compares to the barrier energy density, one does not expect any submicrosecond behaviour. What seems to happen for large electric field pulses, of duration $\tau_{\mathrm{P}}<\tau_{\mathrm{N}}$, is that the energy barrier is localized close to the surfaces, where a rapid localized twist occurs, on a thickness $d_{\mathrm{E}}$. The surface anchoring energy does not play probably a large role. Its strengh $W_{\mathrm{s}} \sim \frac{1}{2} \frac{K}{L} \phi^{2}$ is characterized by the extrapolation length $L$, which gives the distance overwhich a surface torque from the weak anchoring compares with a curvature torque, for a distortion $\phi$ of the molecular orientation $\mathbf{n}_{\mathrm{s}}$ at the surface. $L$ is known, for « strong » anchoring, to be in the $0.1 \mu \mathrm{m}$ range, and to extend to the $\mu \mathrm{m}$ range for weak anchoring. In this case, $L$ compares with $d$, and the weak surface energy does not change significantly the dynamics of the effect. We can estimate the thickness of the surface layer $d_{\mathrm{E}}$ by saying that, for fixed strong anchoring, the curvature energy density associated with $d_{\mathrm{F}}$ must compare with the ferroelectric energy density -- E.P. This result into $E P \sim K / d_{\mathrm{E}}^{2}$ so that the iesponsi: time for large pulses is simply $\tau_{\mathrm{p}} \sim \eta / E P$. To get into the $\tau(\sim 1 \mu \mathrm{s})$ range, one has to ncreas $\epsilon E / E_{\text {th }}$ as $\tau_{\mathrm{N}} / \tau$. One does observe [5] a $E^{-1}$ response time, but also a $E^{-2}$, which is not explained by this model. Anyway, because of the weak anchoring, the response time is purely nematic-like. One would obtain the same short response time with a standard nematic distorted on a short distance $d_{\mathrm{E}}$. The short response time is not related to the volume potential barrier. This makes difficult the multiplexing of a display with $\mathrm{SC}^{*}$, which appears to present a very weak apparent threshold at DC. For very low frequencies, this threshold can even go to zero, in presence of surface disclination lines, which allow for a surface reorientation whithout any jump above the elastic potential barrier. To improve the multiplexing it has been suggested to stabilize the unwound $\mathrm{SC}^{*}$ helix by external $\mathrm{AC}$ electric fields [6]. In practice this would increase the power consumption of electrooptical devices, which seems an important drawback. Finally, the present use of the rapid SC* valve is complicated by all the surface states associated with the polar anchoring. The mastering of surface treatment to induce the right weak surface orientation is now far from complete. An intensive work is curently under way in many applied laboratories to solve these problems.

\section{The surface induced fast instabilities of nematics} (SIFI).

We have recently observed in Orsay [3] polar surface instabilities under the action of an electric field on cyanobiphenyl compounds. The molecules are ali- gned in the homeotropic geometry, i.e. $\mathbf{n}$ is normal to the electrodes. This surface orientation is obtained using a DMOAP silane treatment. A surface polarization can exist at a nematic-solid interface, because the inversion symetry of the bulk material is broken. The origin of this surface polarization can be a permanent polarization of the silane, the polarization associated with the gradient of order parameter at the interface, or simply the flexoelectric polarization. This surface polarization $\mathbf{P}_{\mathbf{S}}$ is coupled with $\mathbf{n}$ so that an applied field opposite to $\mathbf{P}_{\mathbf{S}}$ can induce a molecular surface tilt (Fig. 4). In practice, because of the large dielectric anisotropy of the material, this mechanism cannot lead to high contrast instabilities and seems not usefull for practical application. In addition to this polar surface instability, which appears optically to draw a chart of the variation of the surface anchoring energy on the electrodes, we have observed localized instabilities, of disk-like shape of diameter $\sim 50-200 \mu \mathrm{m}$. At low frequencies $(<1 \mathrm{kHz})$ these points are also excited by a localized polar mechanism, which could be unipolar charge injection, resulting in a vortex flow which would tilt the director $\mathbf{n}$ and result into the large observed contrast. Some of these points can be excited in AC up to one kilohertz, others up to one megahertz with field $\sim 1 \mathrm{~V} / \mu \mathrm{m}$. We do not know now the exact mechanism of these surface induced fast instabilities (SIFI) at high frequencies. To check the response of the SIFI we have measured the amplitude $E_{\mathrm{p}}$ of a rectangular pulse of duration $\sigma_{p}$ which gives the threshold of the effect, or its full contrast (appearance of one fringe in green light). We observe (Fig. 5) an increase of $E_{\mathrm{p}}$ like $\tau_{\mathrm{p}}^{-1 / 2}$. This is characteristic of a nematic like behaviour, with a balance between dielectric and viscous torque $1 / \tau_{\mathrm{p}}=$ $\left(\varepsilon_{\mathrm{a}} / 4 \pi\right) E_{\mathrm{p}}^{2} / \eta$. With $\varepsilon_{\mathrm{a}} \sim 10$, one can explain quantitatively the date of figure 5 . Comparing the SC* bistable display and the SIFI, we can say that both mechanisms present nematic-like response time ; the SIFI gives at room temperature the same response time at full contrast than the $\mathrm{SC}^{*}$ at 60 $70^{\circ} \mathrm{C}$. Keeping in mind that this difference in temperature results in an order of magnitude change for the viscosity we can say that the SIFI is an order of magnitude faster than then $\mathrm{SC}^{*}$ valve.

\section{Conclusion.}

The SC* liquid crystal valve of Clark and Lagerwall is now well known to produce rapid electrooptical switching, using polarized light. It uses a ferroelectric

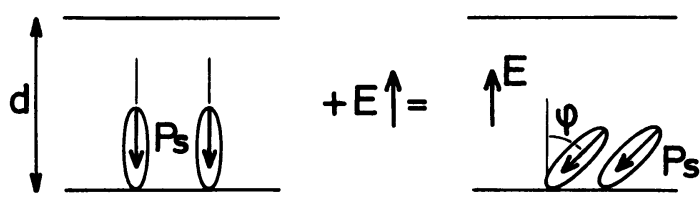

Fig. 4. - Principle of the polar surface instability. $\mathbf{P}_{\mathrm{S}}$ tends to align along $\mathbf{E}$, forcing a surface tilt $\phi$ of the molecules. 


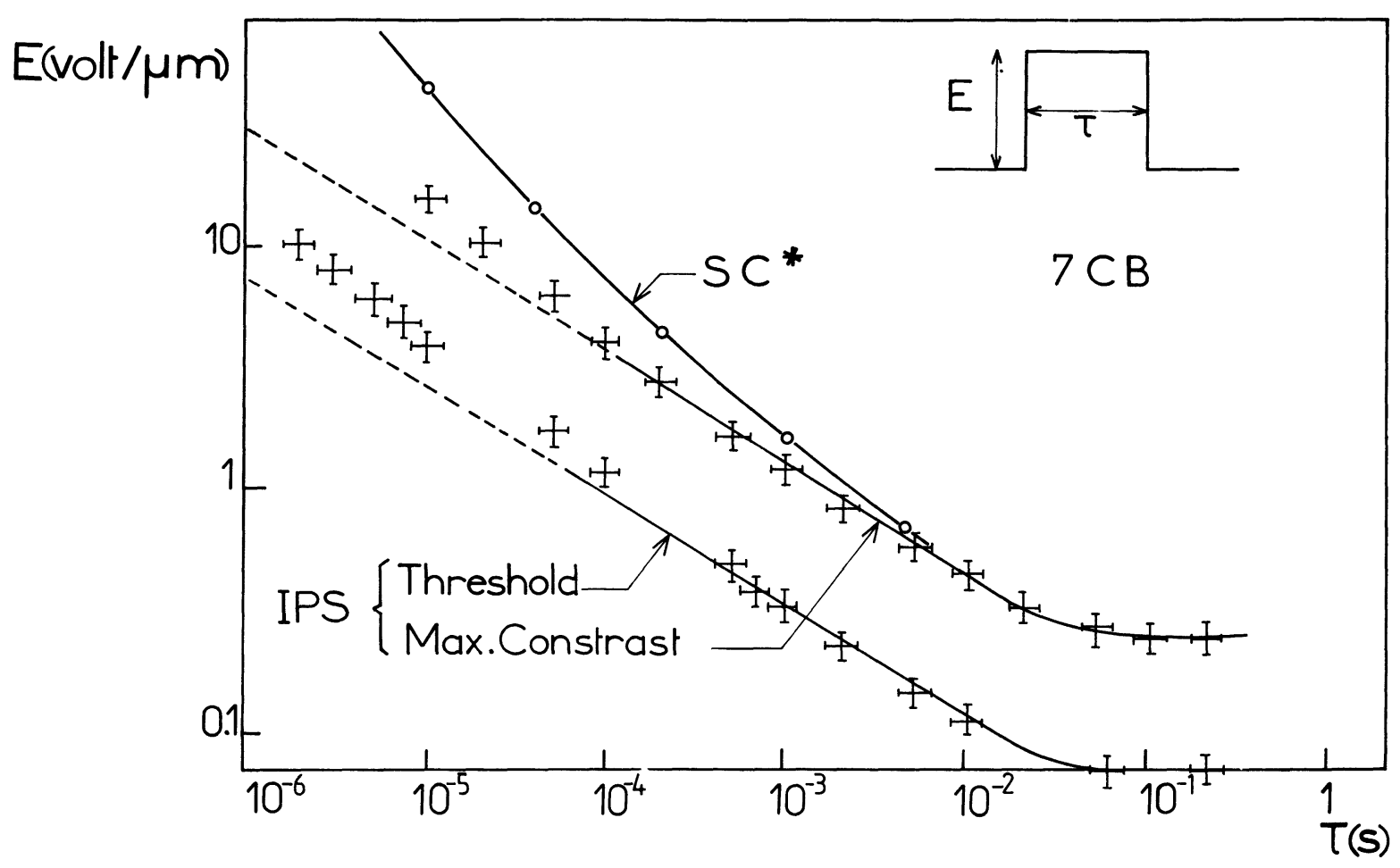

Fig. 5. - Amplitude $E_{\mathrm{p}}$ of an electric field pulse of duration $\tau$, for threshold and full contrast, for SIFI at room temperature. To compare, the corresponding values for the $\mathrm{SC}^{*}$ valve are represented at $68^{\circ} \mathrm{C}$. For short pulse, the SC* valve needs an order of magnitude larger excitation.

property which allows a highly sensitive linear coupling with an external electric field. It also necessitates weak surface anchoring. Its behaviour is not completely understood. It seems to present little or no threshold, the switching being related to the height of a low potential barrier. Its rapid dynamics is probably controlled by a thin elastic surface layer breakdown. Its use is complicated by the existence of many surface states, connected with surface polar anchoring. Materials with a reasonably large range of temperature, with an acceptable tilt angle and a low enough viscosity at room temperature seem to exist, at least in the form of mixtures. Multiplexibility of the SC* is not a solved question. A new surface induced fast instability (SIFI) in nematics has just been discovered in Orsay. It can give the same contrast as the $\mathrm{SC}^{*}$ valve. It has a short response time, for high field, up to the $\mu$ s range. Its response to field pulses can be an order of magnitude faster than the one of a $\mathrm{SC}^{*}$, at room temperature. Its mechanism is not yet clear, giving polar effects at low frequencies and non polar at high frequencies. If we can control its mechanism, the SIFI could become usefull for fast electrooptical switching. Note finally that for both systems the rapid switching effects are related to the high applied field and to the liquid crystal-solid electrode interface properties. Future progress in rapid electrooptic switching implies at better understanding of interface anchoring properties.

\section{Acknowledgments.}

I thank M. Monkade, Ph. Martinot-Lagarde, B. Barbero and J. F. Palierne for interesting discussion and partial disclosure of their results before publication.

\section{References}

[1] For a general description of liquid crystals see : the Physics of Liquid crystals, P. G. de Gennes, Oxford (1974).

[2] Clark, N., Lagerwall, S., Ferroelectrics 59 (1984) 25. In fact, this special issue of Ferroelectric gives a good review of the physics and chemistry of $\mathrm{SC}^{*}$.

[3] Monkade, M., Martinot-Lagarde, Ph., Durand,
G., Europhys. Lett. 2 (1986) 299.

[4] HandsChy M., ClaRK, N., Ferroelectrics 59 (1984) 69.

[5] Clark, N., Handschy, M., Lagerwall, S., Mol. Cryst. Liq. Cryst. 94 (1983) 213.

[6] Le Pesant, M., Dubois, J. C., Mourey, B., Hareng, M., Decobert, G., Proceedings of the Eurodisplay Conference, Paris 1984. 